\title{
PROPOSTA DE DESENVOLVIMENTO DE INOVAÇÃO TECNOLÓGICA EM ARRANJOS PRODUTIVOS LOCAIS
}

\section{PROPOSAL OF THE DEVELOPMENT OF TECHNOLOGICAL INNOVATION FOR PRODUCTIVE ARRANGEMENTS BASED ON INNOVATION TOOLS}

\author{
Adriana Valélia Saraceni ${ }^{1}$; Pedro Paulo de Andrade Junior ${ }^{2}$; Luis Mauricio Resende ${ }^{3}$ \\ ${ }^{1}$ Universidade Tecnológica Federal do Paraná - UTFPR \\ avsaraceni@hotmail.com \\ ${ }^{2}$ Universidade Tecnológica Federal do Paraná - UTFPR \\ pedropaulo@utfpr.edu.br \\ Luis Mauricio Resende \\ ${ }^{3}$ Universidade Tecnológica Federal do Paraná - UTFPR \\ lmresende@utfpr.edu.br
}

\begin{abstract}
Resumo
Este artigo teve como objetivo central evidenciar as principais ferramentas para o desenvolvimento da inovação tecnológica e analisar propostas de desenvolvimento de inovação em Arranjos Produtivos Locais (APL's) a partir destas ferramentas. Como metodologia, do ponto de vista dos seus objetivos, esta pesquisa pode ser considerada como exploratória e descritiva, pois envolve levantamento bibliográfico e análise de exemplos que estimulem a compreensão e pode ser considerada descritiva por descrever as características de levantamento de dados e estabelece relações entre as variáveis. A abordagem do problema acontece de maneira qualitativa, pois o processo e seu significado são os focos principais de abordagem. Alguns estudos sobre ferramentas de inovação tecnológica, e sobre a importância do grau de maturidade para desenvolvimento de inovação foram utilizados como método de análise para se levantar a possibilidade de aplicação. Através da metodologia e do referencial teórico utilizado, verificou-se que as duas principais ferramentas de inovação tecnológica abordadas neste trabalho, demandam uma interação entre empresas e entre diversos atores, no sentido de obterem aumento da competitividade perante o mercado, evidenciando-se assim que em um APL, há uma maior facilidade para a difusão dessas medidas.
\end{abstract}

Palavras-chave: Ferramentas de inovação tecnológica; Arranjos Produtivos Locais; Inovação; Competitividade.

\section{Introdução}

O processo de inovação quando realizado de modo contínuo favorece uma empresas a conseguir estabelecer vantagens competitivas perante os seus concorrentes.

O desenvolvimento e a difusão de novas tecnologias tem um importante papel para o crescimento produtivo, mas de acordo com o entendimento Manual de Oslo (2004), "o processo de 
inovação e seu impacto econômico ainda é deficiente". Nota-se a revolução tecnológica atual na economia mundial frequentemente reconfigurada por conta do surgimento de novas tecnologias da informação e diversas mudanças em campos variados. Mas essas significativas alterações tecnológicas não estão refletindo em melhorias na produtividade e no crescimento da produção (MANUAL DE OSLO, 2004).

Prahalad e Ramaswamy (2003) argumentam que a pressão para criar valor é crescente, a concorrência é intensa, e margens de lucro estão cada vez menores. As receitas tradicionais para práticas de redução de custos como reengenharia e terceirização de serviços já não são suficientes. A necessidade de inovar é maior do que nunca (PRAHALAD e RAMASWAMY, 2003).

Identificar o grau de inovação auxilia as empresas em avaliar o que se deve fazer para estimular a inovação, resultando na construção de crescimento econômico, por isso a importância de empresas inovadoras em identificar sua capacidade de inovação (WEERAWARDENA, 2003; REJEB et al, 2008; NIDUMOLU et al, 2009; WONGLIMPIYARAT, 2010).

Conforme Cassiolato et al (2000), a dimensão territorial é um elemento importante para um APL, pois a proximidade das empresas possibilita o compartilhamento de visões e valores econômicos, bem como vantagens competitivas do produto final.

Os Arranjos Produtivos têm sua estrutura base na criação de uma rede de transações entre atores produtivos e as transações não precisam necessariamente ser ligadas a um bem físico, ou seja, pode englobar atividades de cooperativas, como desenvolvimento de pesquisas em conjunto, pois a organização em rede fomenta o desenvolvimento de relações onde o comportamento dos agentes estabelece interações (VILPOUX e OLIVEIRA, 2010; MASQUIETTO et al, 2011). Os APL's mostram superioridade e impacto na competitividade por facilitar a difusão de informações e características da regionalidade. A aplicação do conhecimento difundido em um Arranjo Produtivo fortalece a capacidade da geração de inovação.

Neste sentido, este trabalho busca verificar algumas ferramentas conceituadas para o desenvolvimento de inovação tecnológica e a possibilidade de interação dessas ferramentas em um arranjo produtivo local, com propósito de fortalecer o desenvolvimento econômico e regional, o progresso da produção e de gerar um fortalecimento da competitividade para os arranjos produtivos locais.

\section{Metodologia}

Para a realização do presente estudo, foram levantas pesquisas bibliográficas, a partir da revisão de literatura referente às ferramentas para desenvolvimento de inovação tecnológica e referencias sobre arranjos produtivos. A abordagem do problema acontece de maneira qualitativa, 
pois se considera que há uma relação dinâmica entre o mundo real e o sujeito que não pode ser traduzido em números, e não se requer o uso de métodos e técnicas estatísticas.

Para fins de caracterização de Arranjos Produtivos foram pesquisadas referencias do BNDES, e IPEA, e foram utilizados dados do IPEA na demonstração do crescente desenvolvimento dos arranjos produtivos no Brasil.

No sentido de selecionar as ferramentas de inovação tecnológica, utilizou-se o Manual de Oslo (2004) alinhando os principais aspectos ao contexto dos APL's no cenário nacional, para direcionar a proposta da pesquisa sobre promover o desenvolvimento da inovação tecnológica nos APL's.

Para caracterizar o momento de aplicação das ferramentas, foi utilizado o estudo analítico do processo de formação e evolução dos arranjos produtivos regionais de Shaohonga et al (2011). Considerando o momento da formação de redes de inovação no APL, fez-se uma adaptação do modelo de Gestão e Operação de redes de cooperação proposto por Gerolamo et al (2008), no sentido de demonstrar a aplicação das ferramentas no processo de rede circular de inovação.

Do ponto de vista dos seus objetivos, esta pesquisa pode ser considerada como exploratória e descritiva, pois de acordo com Gil (1991), a pesquisa exploratória visa proporcionar uma maior familiaridade com o problema e envolve levantamento bibliográfico e análise de exemplos que estimulem a compreensão, entre outros aspectos. Também pode ser considerada descritiva por descrever as características de levantamento de dados e estabelece relações entre as variáveis.

Portanto, o presente artigo aborda algumas ferramentas para inovação tecnológica e busca correlacionar estas ferramentas para serem configuradas entre as empresas de um Arranjo Produtivo Local.

\section{Arranjos produtivos locais e a capacidade de inovação}

Nos últimos anos, observa-se que a inovação e conhecimento são os elementos mais importantes na competitividade. Recentemente, notam-se estes fatores como premissas de processos interativos locais.

De acordo com Banco Nacional de Desenvolvimento (BNDES, 2000) os APL's são definidos como “[...] um fenômeno vinculado às economias de aglomeração, associadas à proximidade física das empresas fortemente ligadas entre si por fluxos de bens e serviços. A concentração geográfica permite ganhos mútuos e operações mais produtivas [...]”.

O desenvolvimento dos arranjos produtivos locais tem grande importância no cenário nacional como mostra o estudo do IPEA sobre o número de APL's potenciais identificados no Brasil em 2004 de acordo com a Tabela 1: 
Tabela 1 - Número de APLs potenciais identificados no Brasil (Elaborado com base nos dados da RAIS, 2004)

\begin{tabular}{cccccc}
\hline Estados & $\begin{array}{c}\text { Total de } \\
\text { aglomeraçóes }\end{array}$ & $\begin{array}{c}\text { Núcleo de Desenvolvimento } \\
\text { Setorial- Regional }\end{array}$ & $\begin{array}{c}\text { Vetor de } \\
\text { Desenvolvimento Local }\end{array}$ & $\begin{array}{c}\text { Vetor } \\
\text { Avançado }\end{array}$ & Embrião \\
\hline SP & 72 & 18 & 15 & 16 & 23 \\
MG & 80 & 17 & 15 & 25 & 23 \\
RS & 63 & 11 & 7 & 35 & 10 \\
SC & 53 & 12 & 2 & 30 & 9 \\
PR & 61 & 9 & 8 & 30 & 14 \\
RJ & 34 & 5 & 3 & 21 & 5 \\
CE & 19 & 7 & 3 & 4 & 5 \\
BA & 53 & 9 & 14 & 14 & 16 \\
PE & 36 & 3 & 3 & 24 & 6 \\
GO & 30 & 3 & 2 & 17 & 8 \\
ES & 25 & 5 & 1 & 17 & 2 \\
PA & 15 & 2 & 1 & 9 & 3 \\
MA & 15 & 1 & 1 & 11 & 2 \\
PI & 9 & 0 & 0 & 5 & 4 \\
TO & 10 & 0 & 0 & 9 & 1 \\
AL & 11 & 3 & 0 & 7 & 1 \\
SE & 16 & 3 & 0 & 7 & 6 \\
AM & 20 & 0 & 0 & 20 & 0 \\
RO & 21 & 1 & 1 & 16 & 4 \\
AC & 5 & 0 & 0 & 4 & 1 \\
AP & 4 & 0 & 0 & 4 & 0 \\
RR & 0 & 0 & 0 & 0 & 0 \\
MT & 39 & 0 & 5 & 13 & 20 \\
MS & 29 & 0 & 0 & 17 & 9 \\
RN & 22 & 1 & 0 & 11 & 8 \\
PB & 20 & 3 & & & 2 \\
\hline
\end{tabular}

Fonte: IPEA- relatório consolidado (2006)

De acordo com o IPEA (2006) os embriões de APL apresentam atrativos para as medidas de políticas e ações institucionais que visem promover a criação e difusão de capacitações entre os produtores locais, com efeitos positivos para a competitividade como a geração de inovação. A tabela 1 apresenta somente o número de APL's potenciais em cada estado conforme cada tipo de aglomerado, mas que os embriões apresentam perspectivas de desenvolvimento.

Os aglomerados produtivos, em maioria, são constituídos por empresas de pequeno porte e é determinante a cooperação e as ações conjuntas, pois essa parceria busca compensar sua dificuldade de competir com empresas maiores. Portanto, tal cooperação tem o potencial de gerar aumento da capacidade produtiva, otimizar custos e aumentar da capacidade de inovação, que possibilitam uma melhoria para enfrentar a competitividade (PETTER et al, 2010).

É importante a maturidade dos aglomerados produtivos já que o foco destes é voltado para produtividade e diversos exemplos da prática posicionam que esta pode ser alavancada com a maturidade. Mas a produtividade não é o único foco dos APL’s, nota-se crescente preocupação com 
a qualidade de vidas dos funcionários, parceria com as instituições de pesquisa e desenvolvimento. Além disso, o investimento em inovação, responsabilidade social e ambiental demostram crescente importância nos arranjos produtivos (PETTER et al, 2010).

Em razão do crescente aumento de competitividade e com clientes cada vez mais seletivos, a PeD demanda alto investimento. Portanto, a fim solucionar essa questão, é fundamental que se desenvolva constantemente um comportamento empreendedor. Sendo assim, percebe-se que os blocos de empresas aliadas para a construção de inovações demostram maiores chances de estabelecer vantagens competitivas (ROCHA, 2004).

Atualmente, os Arranjos Produtivos mostram superioridade e impacto na competitividade para a economia regional. Nota-se que as indústrias que ganham vantagem competitiva, possuem muitas características de regionalidade e estão inseridas em algum complexo industrial (SHAOHONGA et al, 2011).

As inovações geram novos mercados e transformam setores industriais. Estas transformações tem impacto importante nas expectativas de clientes proporcionando maiores ganhos de competitividade (CARLOMAGNO e SCHERER, 2009). Portanto, a maturidade do Arranjo Produtivo consolida uma cooperação com propósitos comuns, que tem o potencial de gerar aumento da capacidade produtiva, otimizar custos, que possibilitam uma melhoria para enfrentar a competitividade e aumentar a capacidade de inovação.

\section{Inovação}

De acordo com Carlomagno e Scherer (2009), nas atuais exigências mundiais que implicam qualidade total, defeito zero e padronização, as práticas gerenciais buscam eliminar erros. Inovar implica em tentativas, buscas, cometer erros e refazer, portanto, neste ambiente onde se busca a previsibilidade, o espaço para a criatividade e para a inovação se tornam restringidos.

A inovação tecnológica é um elemento capaz de gerar de mudanças com questões de ordem técnico-científica, de dimensões de ordem política, econômica e sociocultural. Além disso, é possível encontrar diferenças entre as diversas experiências no que se refere a graus de desenvolvimento, de integração da cadeia produtiva, de articulação e interação entre agentes e instituições locais, e de capacidades sistêmicas para a inovação. Portanto, inovar em países que estão em desenvolvimento necessita de muito planejamento, pois não é uma tarefa simples (CASAGRANDE, 2004; IPEA, 2006).

A formação de competências em inovar e a sua transformação em resultados fazem parte da estratégia da empresa. A estratégia, hoje, é vista como sendo muito mais do que um simples plano: é uma perspectiva, um curso de ação, um "jeito de jogar o jogo" - e de vencê-lo. Estratégias tradicionais são voltadas à manutenção do status quanto ao crescimento incremental. Estratégias 
baseadas na inovação são dirigidas para resultados superiores, para a liderança no lançamento de novas soluções para o mercado (CARLOMAGNO e SCHERER, 2009).

A visão sistêmica da inovação tem ênfase na importância da transferência e difusão de "ideias, habilidades, conhecimentos, informações e sinais" diversos. De acordo com essa visão, os canais e redes que proporcionam a circulação dessas informações estão inseridos em um contexto social, político e cultural, fortemente direcionados e ou limitados pela estrutura institucional (MANUAL DE OSLO, 2004).

A abordagem sistêmica da inovação leva as políticas a enfatizar a interação entre as instituições, para que os processos interativos sejam conduzidos na criação, difusão e aplicação do conhecimento. Deste modo percebe-se o papel indeclinável dos governos para monitorar e organizar a estrutura geral (MANUAL DE OSLO, 2004).

No processo de inovação o conhecimento tácito tem um papel fundamental, já que provém do conhecimento das pessoas, e a socialização do conhecimento dentro da empresa tem o significado de valorizar o ser humano e qualificá-lo para que sua criatividade, iniciativa e seus resultados possam ser fortalecidos. As empresas que visam o capital humano como custos, diminuem sua capacidade de inovar (CARLOMAGNO e SCHERER, 2009).

Prahalad e Ramaswamy (2003) propõem uma diferenciação entre a inovação tradicional e a inovação através de experiência e cooperação. Para os autores, o "valor cada vez mais tem que ser cocriado ${ }^{1}$ com os consumidores, e inovação deve ser focado em sua cocriação de experiências" ${ }^{2}$. Para Prahalad e Ramaswamy (2003), o valor de uma criação é definido pela experiência de um consumidor específico, em um local e tempo específicos em uma determinada situação. Os mesmos autores abordam que no espaço de experiência, um evento desencadeia uma experiência de cocriação, de modo que tal situação tem um contexto no espaço e no tempo, e o envolvimento do indivíduo (consumidor) tem nessa experiência. O significado pessoal derivado da experiência de cocriação é o que determina o valor para o indivíduo.

As atividades de inovação tecnológicas de produtos e de processos (TPP) "são todas as etapas científicas, tecnológicas, organizacionais, financeiras e comerciais que de fato levam, ou pretendem levar, à implantação de produtos ou processos tecnologicamente novos ou aprimorados. Algumas delas podem ser inovadoras por si mesmas, outras, embora não sejam novidades, são necessárias para a implantação" (MANUAL DE OSLO, p. 23, 2004).

\footnotetext{
${ }^{1} \mathrm{O}$ termo cocriação começou a ser usado em 2004 a partir do livro "O futuro da Competição" escrito por C. K. Prahalad e Venkat Ramaswamy, que disseminou o conceito mundialmente. Cocriação é um conceito de marketing e negócios, uma forma de inovação que acontece quando as pessoas de fora da empresa como fornecedores, colaboradores e clientes associam-se com o negócio ou produto agregando inovação de valor, conteúdo ou marketing, e recebendo em troca os benefícios de sua contribuição, sejam eles através do acesso a produtos customizados ou da promoção de suas ideias.

${ }^{2}$ Value will increasingly have to be co-created with consumers, and innovation must be focused on their co-creation experiences.
} 
De acordo com Casagrande (2004), a atividade de criação de produtos tem foco exclusivo no lucro, de modo que relata que "os produtos são desenvolvidos apenas para se adequarem às exigências estético-culturais e condições técnico-econômicas da produção industrial de uma sociedade".

A ideia proposta por Andrew e Sirkin (2003), relaciona a inovação com ganhos financeiros. Segundo os autores, há uma diferença importante entre ser inovador e ser uma empresa inovadora: o primeiro gera um monte de ideias, este último gera muito dinheiro.

Gerenciar o conhecimento nas empresas traz benefícios na atual perspectiva de inovação, bem como a inserção de novos padrões de qualidade e funcionalidade é fator primordial para a organização fique ativa no mercado e perante concorrência (SILVA et al, 2009).

Seja qual for à abordagem da inovação, ela compõe estrutura de gestão abrangente que ajuda a transformar ideias em retornos financeiros para que as empresas ao lançar novos produtos ou serviços possam aplicar melhorias a estes, ou explorar novas oportunidades de negócios e tecnologias, que consequentemente promovem o desenvolvimento social e econômico.

\section{O desenvolvimento da inovação tecnológica}

Uma importante ferramenta para a inovação tecnológica de acordo com Rocha (2004) são as alianças estratégicas, pois esta é um componente da condução de uma empresa para que venha se posicionar melhor no futuro, uma vez que, a partir da estratégia, a empresa poderá selecionar os aspectos mais importantes para sua operação, integrando as principais metas, políticas e sequências de ações. Sendo assim, as ferramentas de inovação tecnológica nas organizações, decorrem da utilização de ferramentas de previsão e de ações de PeD (ROCHA, 2004).

De acordo com Carlomagno e Scherer (2009), “a empresa inovadora tem uma estrutura orgânica, flexível e com poucos níveis hierárquicos. A inovação se origina do caos organizado". Mas a cultura inovadora necessita de modificação de atitudes, pois inovar tem que trazer resultados. Uma empresa pode possuir novos conhecimentos, mas se não os aplicam, não promove resultados, “o segredo não é ser inovativo, é ser inovador".

Inovar demanda diversos fatores que deixam claro que esta não é uma trajetória simples, pois dispor tempo e recursos para resultados nem sempre imediatos dificulta tentativa onde é fundamental tentar novas soluções, e nunca desistir (CARLOMAGNO e SCHERER, 2009).

Ressalta-se que as empresas que não forma alianças estratégicas, tendem a ter maiores dificuldades em se destacar, além de que os clientes tendem a ser tornarem cada vez mais seletivos, principalmente diante do contexto social que valoriza as necessidades de qualidade, sustentabilidade e social, além de outras mudanças comportamentais decorrentes do desenvolvimento dos mercados (ROCHA, 2004). 
As atividades de inovação tecnológica necessitam de investimentos para pesquisa e desenvolvimento que nem sempre resultam em ganhos. O risco de não se obter retorno dos investimentos inibe as atividades inovadoras uma vez que continuar fazendo o habitual não implica. O desenvolvimento de um novo produto ou processo costuma ser longo, de modo que estratégias de curto prazo dificultam que a empresa vise o futuro (CARLOMAGNO e SCHERER, 2009).

Embora a formação e desenvolvimento da rede de inovação seja em uma única empresa, seja em um processo auto organizável e auto dependente, o governo tem um papel importante pois o deve proporcionar um ambiente de cultura industrial, e um mecanismo favorável para que se possa efetivar a capacidade de cooperar e inovar (SHAOHONGA et al, 2011).

O Brasil, com a entrada em vigor da Lei 10.973 de Inovação Tecnológica (LIT) de 2 de dezembro de 2004, passou a contar com um novo instrumento de fomento à inovação e à pesquisa científica e tecnológica no ambiente produtivo, com vistas à capacitação e ao alcance da autonomia tecnológica e ao desenvolvimento industrial do País, sendo uma importante ferramenta para o processo de inovação tecnológica (MATIAS-PEREIRA e KRUGLIANSKAS, 2005).

A criação de valor voltada para o crescimento rentável quase que exclusivamente decorre da inovação (PRAHALAD e RAMASWAMY, 2003).

Com o estímulo da inovação, aumentam se a disponibilização de produtos e serviços mais competitivos no mercado, a geração de emprego é favorecida, com impactos diversos na economia.

Porém, de acordo com Prahalad e Ramaswamy (2003), as empresas começam a perceber que nem o valor nem a inovação podem mais ser sinônimos de sucesso se o foco da empresa estiver apenas nos produtos e nos serviços. Uma nova percepção se faz necessária, que enfoque os clientes de forma individual, fornecendo uma oportunidade de consumo diferenciado, criando um valor exclusivo.

As empresas costuma aumentar a variedade de produtos como estratégia de defesa da concorrência em um espaço cada vez mais sem fronteiras e um espaço competitivo continuamente em transformação. As empresas podem criar novos produtos e expandir seus mercados, em contrapartida, o consumidor pode fazer sua própria combinação na escolha de produtos e serviços a fim de satisfazerem suas necessidades. No entanto, para a maioria dos consumidores, o aumento das características e funções nos produtos, e a diversidade, também pode gerar dificuldade de escolha. Para a maioria dos consumidores, a variedade de produtos não resulta necessariamente em melhores experiências de consumo (PRAHALAD e RAMASWAMY, 2003).

Buscar competitividade através da variedade de produtos ofertados decorre da premissa de que as empresas geram valor através dos produtos e serviço que oferecem o que por sua vez leva a uma visão centrada de inovação. Com o aumento de consumidores cada vez mais informados e de um cenário competitivo em contínua mudança, surgem desafios para essa visão. É 
significativamente mais fácil criar uma variedade de produtos. Mas competir de maneira eficaz apenas pela variedade de produtos já não.

Uma das formas para se ampliar o desenvolvimento de inovações, destacam-se as incubadoras de empresas que facilitam o acesso à infraestrutura, recursos humanos, com novas empresas. A Lei da Inovação Tecnológica busca regularizar a situação das empresas privadas dentro das universidades e reconhece a importância de transformar CeT e PeD em novos produtos e processos ou aprimorar os existentes, visando que produtos finais atendam as expectativas dos mercados nacional e internacional (MATIAS-PEREIRA e KRUGLIANSKAS, 2005).

Os três eixos principais da Lei de Inovação Tecnológica está organizada em torno da constituição de ambiente que favoreça a parceria entre as universidades, institutos tecnológicos e empresas; o estímulo à participação de instituições de ciência e tecnologia no processo de inovação; e o incentivo à inovação na empresa (LEI n. 10.973; MATIAS-PEREIRA e KRUGLIANSKAS, 2005).

As alianças estratégicas entre empresas são importantes para que a construção do processo de inovação tecnológica aconteça de maneira simples e efetiva (ROCHA, 2004).

De acordo com a pesquisa realizada por Matias-Pereira e Kruglianskas (2005), há algumas dúvidas a consistência da Lei de Inovação Tecnológica, que principalmente deve funcionar como instrumento de suporte o ciclo vicioso da dependência tecnológica no Brasil seja rompido.

Destaca-se que a Lei de Inovação Tecnológica, quando busca estabelecer um diálogo entre as instituições produtoras de bens de conhecimento e as produtoras de bens e serviços, emerge mais como um instrumento para facilitar a criação de uma cultura de inovação no País. Entretanto, por si só não é suficiente para mudar a realidade existente nesse campo. Apesar dos instrumentos serem disponibilizados, é necessário haver um maior envolvimento por parte dos atores envolvidos (MATIAS-PEREIRA e KRUGLIANSKAS, 2005).

Para Matias-Pereira e Kruglianskas (2005) a Lei de Inovação Tecnológica decorreu da discussão focada nos recursos e atingiu os aspectos institucionais que agora precisam ser implementados. Os autores consideram que é importante preservar a identidade dos atores, como das universidades e das indústrias e empresas visando que a lei seja um instrumento relevante de fomento às políticas industrial e tecnológica brasileiras.

Vale ressaltar que em 15 de dezembro de 2010 entra em vigor a lei $\mathrm{n}^{\circ} 12.349$, onde passam a vigorar algumas alterações na lei 10.973, de 2 de dezembro de 2004.

Para implantar a Lei de Inovação Tecnológica é importante que ocorra uma efetiva interação das ações governamentais com o segmento privado, a comunidade científica e tecnológica e os trabalhadores. Percebe-se que a Lei de Inovação Tecnológica é um instrumento institucional 
importante no apoio das políticas tecnológicas e industriais em âmbito nacional (MATIASPEREIRA e KRUGLIANSKAS, 2005).

Para aplicar ambas as ferramentas de inovação tecnológica, ao buscar o desenvolvimento, percebe-se a importância da interação de diversos atores como pesquisadores, grupos sociais, indivíduos, empresas entre empresas, agentes econômicos e órgãos do governo para que as potencialidades científicas e as necessidades econômicas e sociais obtenham êxito.

\section{Análise e discussão dos resultados}

Quando o processo de inovação se realiza de forma contínua, isso proporciona que a organização possa estabelecer vantagens competitivas perante aos seus concorrentes (ROCHA, 2004).

De acordo com o estudo analítico do processo de formação e evolução dos arranjos regionais baseado na teoria de auto-organização dos sistemas de complexidade e método sinérgico, realizado por Shaohonga et al (2011), a formação do APL passa por diversas fases, que incluem a comunidade empresarial, o arranjo produtivo, e as redes de inovação.

Inicialmente, os aglomerados surgem em uma determinada região. Eles podem atrair e direcionar as empresas daquela localidade. Através da divisão horizontal e vertical autoprodução e auto organizada e do trabalho baseada na especialização, a comunidade empresarial se forma gradativamente.

Em seguida, de forma não linear, os subsistemas passam a se coordenar com os outros, que proporcionará um aumento da maturidade, aumento da eficácia e auto-organização, transformandose de fato em um arranjo produtivo.

E na terceira fase, o arranjos produtivos podem se direcionar para uma rede circular de inovação, sendo um processo auto organizado o que é o resultado do desenvolvimento do APL no estágio mais avançado. A rede circular de inovação regional é um modelo de desenvolvimento ambientalmente sustentável, onde há maiores vantagens criativa e competitiva, além de vitalidade. No processo de implementação, é de fundamental importância à lei interna de evolução dos arranjos produtivos, além da importância de se criar uma condição ecológica favorável na região, ajustar-se ao ambiente externo, e deixar que o mecanismo de auto-organização no APL desempenhe o seu papel, uma vez que o ambiente onde as organizações operam é estruturado por meio de vínculos produtivos e tecnológicos entre as diversas redes de relações (MASQUIETTO et al, 2011; SHAOHONGA et al, 2011).

Portanto, na terceira fase, há a oportunidade de aplicabilidade das ferramentas de inovação tecnológicas nos APL's onde, as alianças estratégicas tem propósito de desenvolver a parceria no sentido de melhorar a capacidade de aprendizados das empresas para efetivar que avanços 
aconteçam, enquanto a Lei de Inovação Tecnológica que tem o objetivo e do papel de "promover e incentivar o desenvolvimento científico, a pesquisa e a capacitação tecnológicas" no sentido de viabilizar o desenvolvimento (ROCHA, 2004; MATIAS-PEREIRA e KRUGLIANSKAS, 2005), como pode ser observado no Quadro 1:

Quadro 1 - Interação das ferramentas de inovação tecnológica no Arranjo Produtivo

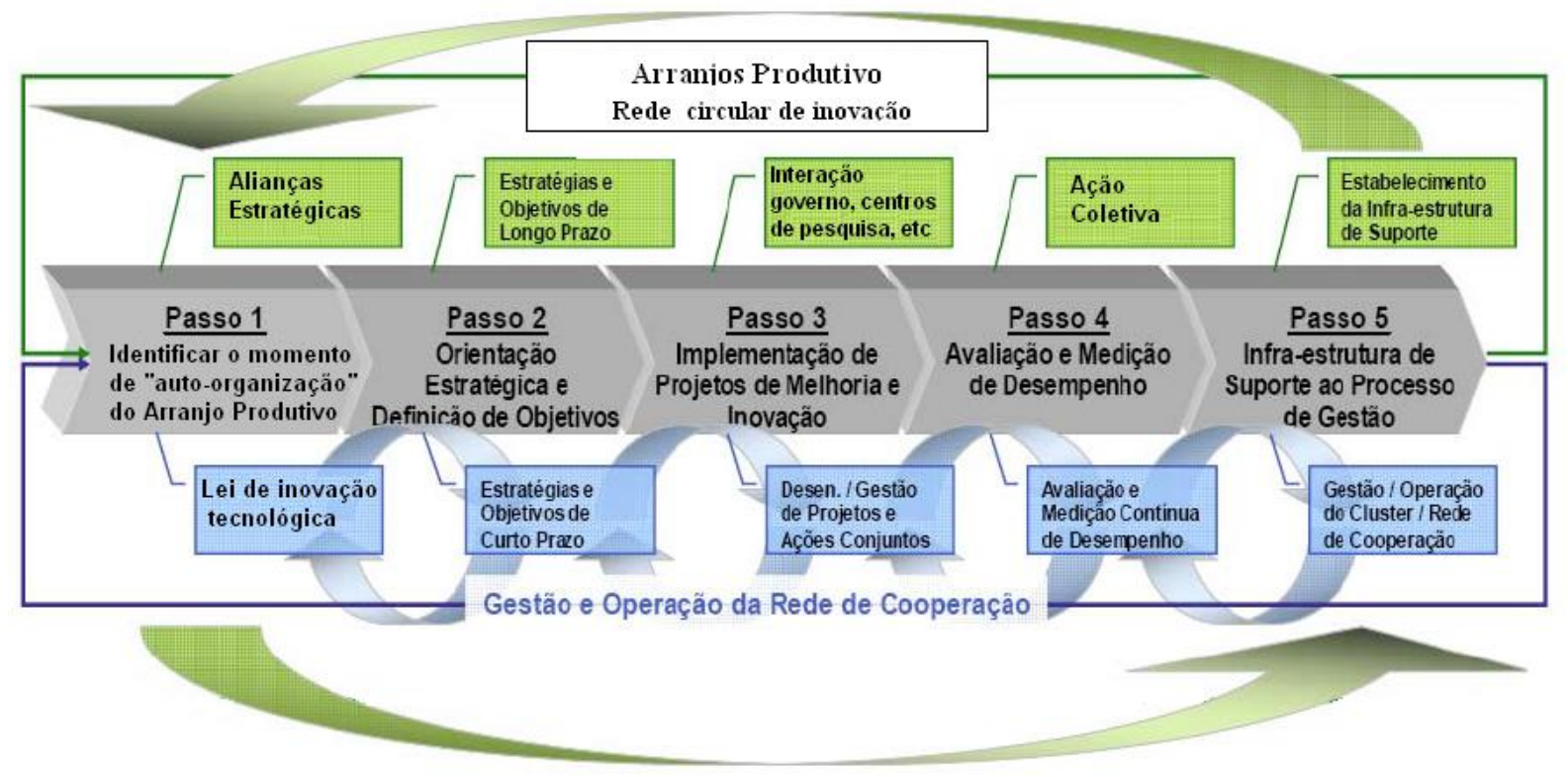

Fonte: Adaptado de Gerolamo et al. (2008)

Por meio de regulamentos sobre ação coletiva e cooperativa de remuneração visando equilibrar o interesse das diversas empresas, o interesse e benefício mútuos podem ser obtidos em cooperação. Somente as redes de inovação criadas a partir de interesse e benefício mútuo podem vir a ter vitalidade duradoura (SHAOHONGA et al, 2011).

A partir da aplicação das ferramentas de inovação tecnológica e da operacionalização com a implantação de métodos e ações cooperadas, o desempenho do processo de inovação do arranjo produtivo tende a se fortalecer. A cooperação seguida do processo de avaliação de desempenho promove o processo de inovação contínua (GEROLAMO et al, 2008).

A Lei de Inovação Tecnológica proporciona a regulamentação enquanto a aliança estratégica promove o fortalecimento da cooperação. Estas ferramentas, ao serem aplicadas nos APL's, proporcionam o aumento da capacidade de inovação e acarretam ganhos econômicos, sociais, ambientais e de competitividade.

\section{Considerações finais}

É importante a construção de formas de coordenação que motivem os sistemas de conhecimento local, fazendo com que o APL se torne um espaço que auxilie no desenvolvimento da 
inovação tecnológica e no aumento da competitividade, pois o desenvolvimento dos arranjos produtivos locais a apontado como aspecto importante de desenvolvimento no cenário nacional.

A inovação possui uma estrutura de gestão abrangente que ajuda a transformar ideias em retornos financeiros para que as empresas possam aplicar melhorias em seus processos, produtos ou serviços.

Para tanto, nota-se a importância da interação de diversos atores como pesquisadores, grupos sociais, indivíduos, empresas entre empresas, agentes econômicos e órgãos do governo para que as potencialidades científicas e as necessidades econômicas e sociais obtenham êxito. A cooperação e um processo de avaliação de desempenho são importantes para promover o processo de inovação contínua, e são aspectos importantes para serem aprofundados em continuidade dos estudos.

Por meio da aplicação das ferramentas de inovação tecnológica e da operacionalização com a implantação de métodos e ações cooperadas, o desempenho do processo de inovação do arranjo produtivo tende a se fortalecer, portanto a cooperação seguida do processo de avaliação de desempenho promove o processo de inovação contínua.

As alianças estratégicas entre empresas são apontadas como uma ferramenta fundamental para o processo de inovação tecnológica, bem como a Lei de Inovação Tecnológica tem importante papel para viabilizar o desenvolvimento científico e de pesquisa.

Ao aplicar estas ferramentas nas empresas pertencentes a um APL's, nota-se o aumento da possibilidade do desenvolvimento da capacidade de inovação, pois no arranjo produtivo há uma maior facilidade para a difusão dessas ferramentas por seu contexto de facilidade das transações entre atores produtivos. Ao ampliar as capacidades de inovação tecnológica em um arranjo produtivo, também se ampliam as possibilidades do desenvolvimento regional, ganhos econômicos, sociais, ambientais e de competitividade.

\section{Abstract}

This article seeks to highlight the main tools for the development of technological innovation looking for the development in Local Productive Arrangements (LPA's) from these tools. In terms of its objectives, this research can be considered as exploratory and descriptive because it involves literature review and analysis of examples that encourage understanding. Also can be considered descriptive, by describing the characteristics of data collection and establishes relationships between variables. The problem approach happens in a qualitative manner, since the process and its meaning are the main focuses. Some studies on technological tools and the importance of LPA maturity level to the development of innovation, are used as a method to analysis of application possibilities. Through the methodology and theoretical framework, it seems that the two major technological tools discussed in this work, require an interaction between companies and between different actors in order to achieve increased competitiveness in the market, thus showing that in a cluster, makes it easier for the dissemination of these measures. 
Key-words: Tools for technological innovation; Local Productive Arrangements; Innovation; Competitiveness.

\section{Referências}

Brasil. LEI No 10.973, DE 2 DE DEZEMBRO DE 2004. Disponível em:

<http://www.planalto.gov.br/ccivil_03/_ato2004-2006/2004/Lei/L10.973.htm>. Acesso em: 02.09.2011.

BNDES Setorial. Características gerais do apoio a arranjos produtivos locais (Por Angela Maria Medeiros M. Santos e Lucimar da Silva Guarneri). Rio de Janeiro, n. 12, p. 197, 2000.

CASSIOLATO, J; LASTRES H. E; SZAPIRO, M. Arranjos e sistemas produtivos locais e proposições de políticas de desenvolvimento industrial e tecnológico. NT 27 - Projeto de pesquisa arranjos e sistemas produtivos locais e as novas políticas. Rio de Janeiro, 2000.

CASAGRANDE, E. F. JR. Inovação tecnológica e sustentabilidade: possíveis ferramentas para uma necessária inferface. Revista Educação e Tecnologia, v. 8, p. 97-109, 2004.

CARLOMAGNO, M.; SCHERER, F. Gestão da Inovação na Prática: como aplicar conceitos e ferramentas para alavancar a inovação. Ed. Atlas, São Paulo, 2009.

GEROLAMO, M. C.; CARPINETTI, L. C. R.; SELIGER, G.; GALDAMEZ, E. V. C. Performance management of regional clusters and SME cooperation networks. International Journal of Business Excellence, v. 1, n. 4, p. 457-483, 2008.

\section{cross ref}

GIL, A. C. Como elaborar projetos de pesquisa. São Paulo: Atlas. 1991.

IPEA - Instituto de Pesquisa Econômica Aplicada. Identificação, mapeamento e caracterização estrutural de arranjos produtivos locais no Brasil. Diretoria de Estudos Setoriais - DISET. Relatório consolidado, 2006.

Manual de Oslo. Proposta de Diretrizes para Coleta e Interpretação de Dados sobre Inovação Tecnológica. OECD, 1997. Rio de Janeiro: FINEP - Financiadora de Estudos e Projetos, 2004.

MASQUIETTO, C. D; SACOMANO N. M; GIULIANI, A. C. Centralidade e densidade em redes de empresas: um estudo no arranjo produtivo local do álcool. Revista de Administração e Inovação, v. 8, n. 1, p. 122-147, 2011.

MATIAS-PEREIRA, J; KRUGLIANSKAS, I. Gestão de Inovação: a lei de inovação tecnológica como ferramenta de apoio às políticas industrial e tecnológica do Brasil. RAE - eletrônica - v. 4, n. 2, Art. 18, 2005.

cross ${ }^{\text {ref }}$

NIDUMOLU, R.; PRAHALAD C. K; RANGASWAMI, M. R. Why sustainability is now the key driver of innovation. Harvard Business Review, 2009.

PETTER, R. R.; RESENDE, L. M.; CERANTO F. A. A.; MARTINS, R. Identificação do nível de maturidade do Arranjo Produtivo Local de Bonés de Apucarana - PR. In: ENCONTRO NACIONAL DE ENGENHARIA DE PRODUÇÃO, 30, 2010. Anais... São Carlos: ABEPRO, 2010.

PRAHALAD, C. K.; RAMASWAMY, V. The new frontier of experience innovation. MIT Sloan Management Review, v. 44, n. 4, p. 12-18, 2003.

REJEB, H. B.; MOREL-GUIMARÃES, L. BOLY, V. ASSIÉLOU, N’DOLI G. Measuring innovation best practices: improvement of an innovation. Technovation, v. 28, 838-854, 2008.

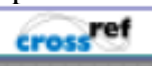

ROCHA, A. C. B. A aliança estratégica como uma ferramenta de inovação tecnológica. In: ENCONTRO NACIONAL DE ENGENHARIA DE PRODUÇÃO, 24, 2004. Anais... Florianópolis: ABEPRO, 2004. 
SILVA, R. J. A.; SICSÚ, A. B; CRISÓSTOMO A. P. Identificação de processos de conhecimento: estudo multicaso em APL de calçados de Campina Grande. Revista Gestão Industrial, v. 05, n.3, s.1, p.189-215, 2009.

\section{cross ref}

SHAOHONGA, C; JIANJUNB, J; QIULANB, X. Research on formation and development of circular industrial clusters and innovative networks. Energy Procedia, v. 5, p. 1519-1524, 2011.

cross ref

VILPOUX, O. F.; OLIVEIRA, E. J. Instituições informais e governanças em arranjos produtivos locais. Revista de Economia Contemporânea, v.14, n.1, 2010.

WEERAWARDENA, J. Exploring the role of market learning capability in competitive strategy. European Journal of Marketing, v. 37, n. 3/4, p. 407, 2003.

cross ref

WONGLIMPIYARAT, J. Innovation index and the innovative capacity of nations. Futures, v. 42, p. 247-253, 2010.

cross ref

\section{Dados dos autores:}

Nome completo: Adriana Valélia Saraceni

Filiação institucional: Universidade Tecnológica Federal do Paraná

Departamento: Engenharia da Produção

Função ou cargo ocupado: Mestranda do Programa de Pós Graduação em Engenharia da Produção PPGEP

Endereço completo para correspondência: Av. Monteiro Lobato s/n, Km 04, CEP 84016-210, Ponta

Grossa - PR - Brasil

Telefones para contato: 4299774437

e-mail: avsaraceni@gmail.com

Nome completo: Pedro Paulo de Andrade Júnior

Filiação institucional: Universidade Tecnológica Federal do Paraná

Departamento: Engenharia da Produção

Função ou cargo ocupado: Professor do departamento de Engenharia de Produção

Endereço completo para correspondência: Av. Monteiro Lobato s/n, Km 04, CEP 84016-210, Ponta

Grossa - PR - Brasil

Telefones para contato:

e-mail:pedropaulo@utfpr.edu.br

Enviado em: 28/04/2012

Aprovado em: 22/03/2013 